\title{
FITOSEÍDEOS (ACARI: PHYTOSEIIDAE) ASSOCIADOS A CAFEZAIS E FRAGMENTOS FLORESTAIS VIZINHOS
}

\author{
Phytoseiids (Acari: Phytoseiidae) associated to coffee plantations \\ and adjacent forest fragments
}

\author{
Ester Azevedo Silva ${ }^{1}$, Paulo Rebelles Reis², Maurício Sérgio Zacarias², Patrícia Pádua Marafeli²
}

\begin{abstract}
RESUMO
Existem poucas informações sobre a fauna de ácaros predadores (Phytoseiidae) em ambientes naturais brasileiros adjacentes a agroecossistemas cafeeiros (Coffea spp.) ou sobre a influência que essa vegetação exerce como reservatório de ácaros predadores. Neste estudo, objetivou-se avaliar a diversidade destes organismos em cafeeiros e fragmentos florestais adjacentes. Coletaram-se amostras das espécies Calyptranthes clusiifolia (Miq.) O. Berg (Myrtaceae), Esenbeckia febrifuga (A. St.-Hil.) A. Juss. ex Mart. (Rutaceae), Metrodorea stipularis Mart. (Rutaceae) e Allophylus semidentatus (Miq.) Radlk._(Sapindaceae), em oito fragmentos florestais, de 5 a 51 ha, e cafezais adjacentes, nos meses de junho (final período chuvoso) e outubro (final período seco) nos anos 2004 e 2005, na região Sul do Estado de Minas Gerais. Ácaros foram extraídos das folhas, utilizando o método de lavagem e, em seguida, montados em lâminas de microscopia em meio de Hoyer, para identificação específica. No total foram identificados 2.348 fitoseídeos, sendo 2.090 nos fragmentos florestais e 258 espécimes nos cafezais adjacentes, pertencentes a 38 espécies. Servindo-se de análise faunística, a espécie Iphiseiodes zuluagai Denmark \& Muma, 1972 apresentou os melhores índices no agroecossistema cafeeiro, sendo muito frequente e constante nas épocas estudadas. Nos fragmentos florestais Amblyseius herbicolus Chant, 1959, Iphiseiodes affs. neonobilis Denmark \& Muma, 1978, Leonseius regularis DeLeon, 1965 e Euseius alatus DeLeon, 1966 foram dominantes, muito abundantes, muito frequentes e constantes nas épocas estudadas. Podemos concluir que a vegetação nativa abriga ácaros predadores, inimigos naturais de ácaros-praga, que ocorrem na cultura cafeeira, possibilitando o desenvolvimento de programas de manejo ecológico com áreas de vegetação natural e agroecossistemas cafeeiros adjacentes.
\end{abstract}

Termos para indexação: Acari, manejo de pragas, vegetação nativa, Coffea, cafeeiro.

\section{ABSTRACT}

There is little information about the fauna of predatory mites (Phytoseiidae) in Brazilian natural environments, adjacent to coffee agroecosystems (Coffea spp.), or about the influence exerted by neighbor vegetation as a reservoir of predatory mites. The objective of this study was to evaluate the diversity of these organisms in coffee plantations and adjacent forest fragments. Samples of the species Calyptranthes clusiifolia (Miq.) O. Berg (Myrtaceae), Esenbeckia febrifuga (A. St.-Hil.) A. Juss. ex Mart., Metrodorea stipularis Mart. (Rutaceae) and Allophylus semidentatus (Miq.) Radlk. (Sapindaceae) were collected in eight forest fragments, from 5 to $51 \mathrm{ha}$, adjacent to coffee plantations, in June (end of the rainy season) and October (end of the dry season) in the years of 2004 and 2005, in the Southern region of State of Minas Gerais. Leaf mites were extracted using the wash method, mounted in microscopy slides with Hoyer's medium for identification. A total of 2.348 phytoseiids was collected, being 2.090 in the forest fragments and 258 in adjacent coffee plantations, belonging to 38 species. According to fauna analysis, Iphiseiodes zuluaguai Denmark \& Muma, the year of 1972 presented the best indexes in the coffee agroecosystem, being very frequent and constant in those periods. In the forest fragments, Amblyseius herbicolus Chant, 1959, Iphiseiodes affs. neonobilis Denmark \& Muma, 1978, Leonseius regularis DeLeon, 1965 and Euseius alatus DeLeon, 1966 were dominant, very abundant, very frequent and constant in those periods. One may conclude that the native vegetation shelters predator mite, natural enemies of mite-pests that still occur in coffee culture, making possible ecological management program development involving areas of natural vegetation and adjacent coffee agroecosystems.

Index terms: Acari, pest management, natural vegetation, Coffea, coffee plant.

(Recebido em 17 de abril de 2007 e aprovado em 12 de novembro de 2009)

\section{INTRODUÇÃO}

A manipulação e a alteração humana dos ecossistemas, com o propósito de estabelecer uma produção agrícola, tornam os agroecossistemas muito diferentes dos ecossistemas naturais. Em nenhum outro aspecto da agricultura, as consequencias da redução da biodiversidade são tão evidentes quanto na esfera do manejo de pragas agrícolas. Por isso, um dos motivos mais importantes para manter a biodiversidade dos ecossistemas naturais é dela ser a fonte de todas as plantas e animais utilizados atualmente na agricultura (Altieri, 2002).

O estudo dos organismos presentes na vegetação natural permite a obtenção de conhecimentos fundamentais

\footnotetext{
${ }^{1}$ Universidade Estadual do Maranhão/UEMA - Centro de Ciências Agrárias/CCA - Departamento de Fitotecnia e Fitossanidade - Cidade Universitária Paulo VI - São Cristóvão - Cx. P. 09 - 65055-310 - São Luís, MA - esterazevedo@yahoo.com.br
} 'Empresa de Pesquisa Agropecuária de Minas Gerais/EPAMIG - Lavras, MG 
na área de controle biológico aplicado. Pragas agrícolas podem ser originárias de habitats naturais, onde raramente atingem altos níveis populacionais. O conhecimento dos hospedeiros, ou substratos originais, e do sistema em que estão inseridos também parecem fundamentais na tentativa de reestruturar o ambiente, de forma a torná-lo mais favorável ao desenvolvimento dos inimigos naturais e menos favorável ao desenvolvimento das pragas. As alterações programadas do ambiente podem promover a manutenção de níveis mais adequados de inimigos naturais de pragas nos agroecossistemas (Moraes \& Flechtmann, 2008). Alguns trabalhos já foram desenvolvidos no Brasil sobre ácaros fitoseídeos em ambientes de floresta nativa (Feres \& Moraes, 1998; Lofego et al., 2000; Zacarias \& Moraes, 2001; Daud \& Feres, 2005; Demite \& Feres, 2005, 2007; Castro \& Moraes, 2007; Feres et al., 2009). Porém, pouco se conhece sobre fitoseídeos associados a cafezais e fragmentos florestais adjacentes. Portanto, Neste estudo, objetivou-se conhecer a diversidade de ácaros, em cafeeiros e fragmentos florestais adjacentes, pertencentes à família Phytoseiidae.

\section{MATERIAL E MÉTODOS}

Este trabalho foi realizado em alguns municípios da região Sul de Minas, estado de Minas Gerais, em fragmentos florestais de diferentes tamanhos e cafeeiros adjacentes. Foram realizadas coletas em junho, considerado como final do período chuvoso e, em outubro, considerado final do período seco (Tabela 1), nos anos de 2004 e 2005.

Na região, a paisagem circundante dos fragmentos sofreu intervenção antrópica, para implantação, na sua maioria, de agroecossistemas cafeeiros. Os sistemas cafeeiros, das cultivares Catuaí e Mundo Novo (Coffea arabica L.), com idades variando entre 7 e 20 anos, estavam plantados no sistema tradicional de cultivo e apresentavam áreas variando de 70 ha a 190 ha (Tabela 2). Foram selecionadas oito matas ( 5 a $51 \mathrm{ha}$ ) nativas e adjacentes a esses cafezais, reconhecidas como fazendo parte do sistema Mata Atlântica e estão relacionadas na Tabela 2. A seleção dos fragmentos florestais, adjacentes aos agroecossistemas cafeeiros, foi feita com o uso de imagens Landsat. Em cada fragmento selecionou-se quatro espécies vegetais, comuns a todos os fragmentos, que tiveram material botânico coletado (ramos com folhas e quando possível flores e frutos), para identificação no Herbário da Universidade Federal de Lavras. As espécies estudadas foram: 1) Calypthranthes clusifolia (Miq.) O. Berg, Myrtaceae; 2) Esenbeckia febrifuga (A. St.-Hil.) A. Juss. ex Mart., Rutaceae; 3) Metrodorea stipularis Mart.; Rutaceae e 4) Allophyllus semidentatus (Miq.) Radlk, Sapindaceae (Lorenzi, 2002).

Em quatro espécimes de cada espécie vegetal, distanciadas 20 metros uma das outras, foi realizada a coleta de folhas/folíolos, em caminhamento na região mais central de cada fragmento. A coleta foi feita com auxílio de um podão dotado de cabo telescópico e cordel. Servindo-se da variação específica no tamanho das folhas foram estabelecidas três categorias para determinação do tamanho da amostra por planta: (a) plantas com folhas pequenas -40 folhas amostradas, sendo 20 apicais e 20 medianas (A. semidentatus); (b) plantas com folhas médias - 30 folhas, sendo 15 apicais e 15 medianas $(C$. clusiifolia e E. febrifuga e (c) plantas com folhas grandes 20 folhas, sendo 10 apicais e 10 medianas (M. stipularis).

Considerou-se como plantas de folhas pequenas as que apresentaram folhas de tamanho menor do que 6 $\mathrm{cm}$ de largura e menor do que $10 \mathrm{~cm}$ de comprimento; folhas médias com tamanho entre $6-15 \mathrm{~cm}$ de largura e $10-20 \mathrm{~cm}$ de comprimento e de folhas grandes com tamanho maior que $15 \mathrm{~cm}$ de largura e maior que $20 \mathrm{~cm}$ de comprimento. Sempre que possível e em igual número foram coletadas tanto folhas apicais quanto medianas, sendo as apicais as primeiras cinco folhas a partir do ápice de um ramo e as medianas entre as demais folhas, sendo totalmente formadas e não senescentes.

O material coletado foi acondicionado em sacos plásticos devidamente etiquetados e transportados ao Laboratório de Acarologia da EPAMIG-CTSM/EcoCentro. Foram mantidas em refrigerador (aproximadamente $10^{\circ} \mathrm{C}$ ) até o término do período de coletas (aproximadamente três

Tabela 1 - Temperatura média $\left({ }^{\circ} \mathrm{C}\right)$, precipitação pluviométrica $(\mathrm{mm})$ e umidade relativa $(\%)$, referentes aos períodos que antecederam as coletas, observadas na estação climatológica principal de Lavras (Convênio UFLA/ INMET).

\begin{tabular}{lccc}
\hline \multicolumn{1}{c}{ Período } & Temperatura média $\left({ }^{\circ} \mathrm{C}\right)$ & Precipitação pluviométrica $(\mathrm{mm})$ & U.R $(\%)$ \\
\hline Chuvoso / 2004 (novembro.- maio) & 21,3 & 1.336 & 76,9 \\
Seco / 2004 (junho- outubro) & 17,9 & 153 & 69,2 \\
Chuvoso / 2005 (novembro.- maio) & 21,8 & 1.158 & 75,4 \\
Seco / 2005 (junho- outubro) & 19,1 & 277 & 71,6 \\
\hline
\end{tabular}


dias), sendo então submetidas à lavagem para extração dos ácaros.

Nos cafezais, adjacentes aos remanescentes florestais, foram demarcados três pontos amostrais e, nesses três unidades amostrais, sendo um na borda do cafezal, área de contato com o fragmento, e os outros dois pontos a 25 e $50 \mathrm{~m}$ distantes da borda. Nas quatro amostragens realizadas, duas em 2004 e duas em 2005 foram coletadas 15 folhas do terço superior e 15 do terço mediano de dois cafeeiros, escolhidos ao acaso, em cada unidade amostral, totalizando 180 folhas por cafezal por amostragem, 60 na borda, 60 a $25 \mathrm{~m}$ da borda e 60 a $50 \mathrm{~m}$ da borda. Após a coleta das folhas, utilizou-se o mesmo procedimento adotado para as folhas coletadas dos fragmentos florestais.

A extração dos ácaros das espécies florestais e dos cafeeiros foi realizada pelo método de lavagem das folhas. Esse método foi escolhido entre outros existentes por permitir um maior rendimento na extração de ácaros plantícolas, quando se trata de grande número de amostras (Spongoski et al., 2005), como ocorreu no presente trabalho. Os ácaros coletados foram acondicionados em frascos plásticos, com capacidade aproximada de 30ml, contendo álcool $70 \%$.
Posteriormente, procedeu-se à montagem dos ácaros em lâminas de microscopia com meio de Hoyer (Flechtmann, 1985) para identificação específica dos ácaros. Foram utilizadas chaves dicotômicas e trabalhos de revisão como recursos para identificação dos espécimes de Phytoseiidae encontrados. Foi reservada uma amostra representativa das espécies encontradas (Voucher) para a coleção de referência de ácaros do Laboratório de Acarologia da EPAMIG-CTSM/EcoCentro, Lavras, estado de Minas Gerais. Os cálculos foram realizados com o uso do programa de análise faunística ANAFAU (Lofego \& Moraes, 2006).

\section{RESULTADOS E DISCUSSÃO}

Foi coletado um total de 2.348 espécimes de ácaros da família Phytoseiidae nas quatro épocas de coleta nos anos de 2004 e 2005, sendo 2.090 encontrados nos oito fragmentos florestais e 258 nos agroecossistemas cafeeiros adjacentes, em 38 espécies.

Embora os níveis populacionais das espécies apresentem grande flutuação ao longo do ano e grande variação ano a ano (Feres et al., 2002; Daud \& Feres, 2005; Hernandes \& Feres, 2006; Demite \& Feres, 2007) foi realizada

Tabela 2 - Fragmentos florestais e cafezais estudados quanto à diversidade de ácaros na região Sul de Minas, estado de Minas Gerais, durante os anos de 2004 e 2005.

\begin{tabular}{|c|c|c|c|c|c|c|}
\hline \multirow{2}{*}{ Fazendas } & \multirow{2}{*}{ Proprietário } & \multirow{2}{*}{ Município } & \multirow{2}{*}{ Área (ha) } & \multicolumn{2}{|c|}{ Cafezal } & \multirow{2}{*}{$\begin{array}{c}\text { Coordenadas UTM } \\
\text { Datum WGS } 84^{1}(23 \mathrm{~K})\end{array}$} \\
\hline & & & & Cultivar & Idade (anos) & \\
\hline $\begin{array}{l}\text { Fazenda } \\
\text { Renascer. (II) }\end{array}$ & $\begin{array}{c}\text { Nelson Batista } \\
\text { da Silva }\end{array}$ & Lavras & 5,0 & Catuaí & 10 & $\begin{array}{c}N-7650025 \\
E-490815\end{array}$ \\
\hline $\begin{array}{l}\text { Fazenda } \\
\text { Renascer (I) }\end{array}$ & $\begin{array}{c}\text { Nelson Batista } \\
\text { da Silva }\end{array}$ & Lavras & 9,0 & Catuaí & 7 & $\begin{array}{c}N-7651325 \\
E-492600\end{array}$ \\
\hline $\begin{array}{l}\text { Fazenda Ponte } \\
\text { Alta }\end{array}$ & $\begin{array}{c}\text { Colotário } \\
\text { Figueiredo }\end{array}$ & $\begin{array}{l}\text { Santana da } \\
\text { Vargem }\end{array}$ & 14,0 & Acaiá & 10 & $\begin{array}{c}N-7648323 \\
E-443590\end{array}$ \\
\hline Fazenda Cafua & $\begin{array}{c}\text { Carlos Alberto } \\
\text { Carvalho }\end{array}$ & Ijaci & 18,0 & Catuaí & 12 & $\begin{array}{c}N-7657740 \\
E-500675\end{array}$ \\
\hline $\begin{array}{l}\text { Fazenda } \\
\text { Pinheiros }\end{array}$ & $\begin{array}{c}\text { Raquel Assis de } \\
\text { Andrade } \\
\text { Claudino }\end{array}$ & Coqueiral & 24,0 & $\begin{array}{l}\text { Mundo } \\
\text { novo }\end{array}$ & 7 & $\begin{array}{c}N-7655193 \\
E-452423\end{array}$ \\
\hline $\begin{array}{l}\text { Fazenda Santa } \\
\text { Maria }\end{array}$ & Thalmo Barbosa & $\begin{array}{l}\text { Santana da } \\
\text { Vargem }\end{array}$ & 35,0 & $\begin{array}{l}\text { Mundo } \\
\text { novo }\end{array}$ & 12 & $\begin{array}{c}N-7648741 \\
E-445061\end{array}$ \\
\hline $\begin{array}{l}\text { Fazenda N.S. } \\
\text { Pompeia }\end{array}$ & $\begin{array}{l}\text { Reinaldo } \\
\text { Menberg }\end{array}$ & $\begin{array}{l}\text { Santana da } \\
\text { Vargem }\end{array}$ & 45,0 & $\begin{array}{c}\text { Mundo } \\
\text { novo }\end{array}$ & 10 & $\begin{array}{c}N-7646957 \\
E-445415\end{array}$ \\
\hline $\begin{array}{l}\text { Fazenda do } \\
\text { Trocadeiro }\end{array}$ & $\begin{array}{c}\text { Antonio Aurélio } \\
\text { Chaves }\end{array}$ & $\begin{array}{l}\text { Santana da } \\
\text { Vargem }\end{array}$ & 51,0 & $\begin{array}{l}\text { Mundo } \\
\text { novo }\end{array}$ & 20 & $\begin{array}{c}N-7646115 \\
E-445763\end{array}$ \\
\hline
\end{tabular}

${ }^{1}$ Coordenadas obtidas por GPS 
a análise faunística para os dois anos de estudo, 2004 e 2005 (meses de junho e outubro), não sendo, portanto, resultados conclusivos, mas indicadores da diversidade desses ácaros nessas épocas e nesses anos.

Do total de ácaros fitoseídeos coletados nos agroecossistemas cafeeiros, Iphiseiodes zuluagai Denmark \& Muma, 1972, com 134 espécimes, foi a espécie mais numerosa dentre as que ocorreram em junho e outubro de 2004 e 2005, sendo classificada como superdominante, muito abundante, muito frequente e constante (Tabela 3 ). I. zuluagai foi encontrado em todas as épocas de coleta. Isso indica que esse predador é adaptado ao agroecossistema cafeeiro, na região em estudo, podendo estar contribuindo para o controle biológico natural dos ácaros-praga que ocorrem em cafezais. Pallini Filho et al. (1992) também observou ser I. zuluagai a espécie com maior número de espécimes na região do Sul de Minas, o que sugere ser essa uma espécie importante para a cafeicultura da região. Feres et al. (2005) destaca que $I$. zuluagai foi encontrada em 14 espécies de plantas, em fragmento de mata nativa no estado de São Paulo. Reis et al. (2000), demonstraram o potencial de predação que os ácaros fitoseídeos, encontrados em cafeeiros, apresentam sobre Brevipalpus phoenicis (Geijskes, 1939) (Acari: Tenuipalpidae).

Amblyseius herbicolus (Chant, 1959) e Euseius alatus DeLeon, 1966, espécies de fitoseídeos muito encontradas nos cultivos de cafezais da região (Reis \& Zacarias, 2007), ocorreram em três das quatro épocas de coleta, mostrando-se muito frequentes no agroecossistema cafeeiro. Isso sugere que essa proximidade da vegetação nativa pode estar servindo de reservatório desses ácaros

Tabela 3 - Análise faunística de ácaros da família Phytoseiidae encontradas em cafezais, adjacentes aos fragmentos florestais, em quatro coletas nos anos de 2004 e 2005 na região Sul de Minas, estado de Minas Gerais.

\begin{tabular}{lcccccc}
\hline \multicolumn{1}{c}{ Espécie } & Número de espécimes & Número de coletas & $\mathrm{D}^{1}$ & $\mathrm{~A}^{2}$ & $\mathrm{~F}^{3}$ & $\mathrm{C}^{4}$ \\
\hline Amblyseius acalyphus & 14 & 4 & $\mathrm{D}$ & $\mathrm{Ma}$ & $\mathrm{MF}$ & $\mathrm{W}$ \\
Amblyseius compositus & 9 & 4 & $\mathrm{D}$ & $\mathrm{A}$ & $\mathrm{MF}$ & $\mathrm{W}$ \\
Amblyseius herbicolus & 25 & 3 & $\mathrm{D}$ & $\mathrm{Ma}$ & $\mathrm{MF}$ & $\mathrm{W}$ \\
Amblyseius impressus & 5 & 2 & $\mathrm{ND}$ & $\mathrm{C}$ & $\mathrm{F}$ & $\mathrm{W}$ \\
Amblyseius operculatus & 0 & 0 & $\mathrm{ND}$ & $\mathrm{R}$ & $\mathrm{PF}$ & $\mathrm{Y}$ \\
Amblyseius spiculatus & 1 & 1 & $\mathrm{ND}$ & $\mathrm{R}$ & $\mathrm{PF}$ & $\mathrm{Y}$ \\
Euseius alatus & 22 & 3 & $\mathrm{D}$ & $\mathrm{Ma}$ & $\mathrm{MF}$ & $\mathrm{W}$ \\
Euseius citrifolius & 7 & 2 & $\mathrm{D}$ & $\mathrm{C}$ & $\mathrm{F}$ & $\mathrm{W}$ \\
Euseius concordis & 3 & 3 & $\mathrm{ND}$ & $\mathrm{c}$ & $\mathrm{F}$ & $\mathrm{W}$ \\
Euseius ho & 2 & 1 & $\mathrm{ND}$ & $\mathrm{d}$ & $\mathrm{PF}$ & $\mathrm{Y}$ \\
Iphiseiodes zuluagai & 134 & 4 & $\mathrm{SD}$ & $\mathrm{ma}$ & $\mathrm{MF}$ & $\mathrm{W}$ \\
Phytoseius (Horridus) sp. & 1 & 1 & $\mathrm{ND}$ & $\mathrm{r}$ & $\mathrm{PF}$ & $\mathrm{Y}$ \\
Proprioseiopsis cannaensis & 1 & 1 & $\mathrm{ND}$ & $\mathrm{r}$ & $\mathrm{PF}$ & $\mathrm{Y}$ \\
Proprioseiopsis affs. neotropicus & 4 & 1 & $\mathrm{ND}$ & $\mathrm{c}$ & $\mathrm{F}$ & $\mathrm{Y}$ \\
Proprioseiopsis dominigos & 1 & 1 & $\mathrm{ND}$ & $\mathrm{d}$ & $\mathrm{PF}$ & $\mathrm{Y}$ \\
Typhlodromips manihoti & 1 & 1 & $\mathrm{ND}$ & $\mathrm{r}$ & $\mathrm{PF}$ & $\mathrm{Y}$ \\
Typhlodromips mangleae & 1 & 1 & $\mathrm{ND}$ & $\mathrm{r}$ & $\mathrm{PF}$ & $\mathrm{Y}$ \\
\hline
\end{tabular}

${ }^{1}$ Dominância : SD - superdominante, D - dominante, ND - não dominante.

(Método de Laroca e Mielke).

${ }^{2}$ Abundância : ma - muito abundante, $\mathrm{a}$ - abundante, $\mathrm{c}$ - comum, $\mathrm{d}$ - disperso, $\mathrm{r}$ - raro.

${ }^{3}$ Frequência : $\mathrm{PF}$ - pouco frequente, $\mathrm{MF}$ - muito frequente, $\mathrm{F}$ - frequente.

${ }^{4}$ Constância: W - constante, Y - acessória, Z - acidental. 
predadores. Demite \& Feres (2005) também destacam a importância da vegetação natural vizinha às áreas de cultivo.

Amblyseius acalyphus (Denmark \& Muma, 1973), com 14 espécimes mostrou-se dominante, abundante, muito frequente e constante nos cultivos de cafeeiro. Amblyseius compositus (Denmark \& Muma, 1973) ocorreu em menor quantidade, comparativamente às demais espécies, mas foi encontrada nos dois meses amostrados dos anos de 2004 e 2005. Feres et al. (2005) encontraram as espécies A. acalyphus, A. compositus, Euseius concordis (Chant, 1959), Neoseiulus tunus DeLeon, 1967 e Amblyseius aerialis (Muma, 1955) entre outras, em fragmentos de mata nativa, assim como neste trabalho.

Euseius citrifolius Denmark \& Muma, 1970, em comparação com os demais fitoseídeos que ocorreram nos cafezais, foi considerado dominante, comum, frequente e constante (Tabela 3). Em parte, essas ocorrências já foram relatadas por Pallini Filho et al. (1992) e Reis \& Zacarias (2007), ao observarem ácaros predadores da família Phytoseiidae em cultivo de cafeeiro na região Sul de Minas. Spongoski et al. (2005) destacaram A. herbicolus como sendo comumente encontrado associado ao cafeeiro no Cerrado de Minas Gerais. Isto evidencia a ocorrência natural de ácaros predadores no agroecossistema cafeeiro evidenciando a importância desses ácaros do ponto de vista do controle biológico, levando-se em consideração que os ácaros predadores da família Phytoseiidae são os principais inimigos naturais dos ácaros-praga (McMurtry \& Croft, 1997). E. citrifolius ocorreu, também, na vegetação nativa. Feres \& Moraes (1998) destacaram a predominância dessa espécie dentre as demais encontradas em seringal e ainda foi observada em fragmento florestal adjacente (Demite \& Feres, 2005).

Nos cultivos de cafeeiros, da região sul do estado de Minas, os fitoseídeos I. zuluagai, A. herbicolus, E. alatus e A. acalyphus foram dominantes, abundantes, frequentes e constantes (Tabela 3). Assim, estes predadores podem ser considerados contribuintes relevantes no controle natural dos ácaros-praga que ocorrem na cultura do cafeeiro, e para uma maior eficiência desses ácaros como inimigos naturais deve-se atentar para as formas de manejo a serem utilizadas, que venham propiciar sua conservação e aumento nesses ecossistemas, tomando-se cuidado especial em evitar a utilização de produtos fitossanitários não seletivos aos inimigos naturais, garantindo assim sua sobrevivência nesses ambientes.

Nos fragmentos florestais, adjacentes aos cafezais, os fitoseídeos A. herbicolus (416 espécimes), I. affs. neonobilis (406), L. regularis (257), E. alatus (142) e Euseius ho (DeLeon, 1965), com 102 espécimes mostraram-se as espécies mais numerosas e muito frequentes, ocorrendo nas quatro épocas de coleta, nesses ambientes naturais. Amblyseius operculatus (DeLeon, 1967), com 84 espécimes foi dominante, abundante, muito frequente e constante, enquanto Phytoseius (Horridus) sp., com 69 espécimes, foi comum, frequente e constante nos ecossistemas estudados (Tabela 3).

Nesses fragmentos, as espécies $A$. compositus e $A$. herbicolus alcançaram a mesma classificação encontrada em cafeeiros. Os ácaros comuns nos sistemas cafeeiros e na vegetação nativa adjacente evidenciam a influência desses ambientes naturais como hospedeiros de ácaros predadores que, provavelmente, migram espontaneamente para esses agroecossistemas, e auxiliam no controle natural dos ácaros-praga que ocorrem em cafeeiro. Os ácaros $E$. alatus, E. citrifolius, A. impressus e E. concordis foram espécies que ocorreram nos dois ecossistemas. Pode-se supor que essas espécies podem ser encontradas em maior abundância e com mais frequência em outras espécies florestais não contempladas nesta pesquisa.

I. affs. neonobilis e L. regularis, foram as espécies que mais se destacaram no ecossistema natural, recebendo a classificação de dominantes, muito abundantes, muito frequentes e constantes (Tabela 4). Essas espécies, apesar de não terem sido encontradas em cafeeiros, pela abundância observada nesses ambientes, merecem ser melhor estudadas para possível uso em programas de controle biológico de ácaros-praga. Essas espécies são praticamente desconhecidas em relação à sua ecologia e potencial de predação.

As espécies A. herbicolus, I. affs. neonobilis, $L$. regularis, $E$. alatus e $E$. ho (Tabela 4) foram, de uma forma geral, as mais frequentes nos fragmentos nos meses de junho e outubro, sendo dominantes e muito abundantes. Apesar da predominância desses ácaros em praticamente todos os fragmentos florestais, à exceção de estudos com A. herbicolus e E. alatus (Reis et al., 2000, 2003), muito pouco se sabe sobre os aspectos comportamentais desses ácaros, podendo ser utilizados no controle de pragas atuais ou que possam vir a ser prejudiciais aos agroecossistemas adjacentes no futuro.

Castro \& Moraes (2007), estudando outros ecossistemas naturais destaca a ocorrência expressiva das espécies de ácaros predadores fitoseídeos A. acalyphus e A. compositus, o que demonstra serem essas espécies adaptadas a diferentes tipos de substratos. Lofego \& Moraes (2006) relataram que $E$. citrifolius e $A$. acalyphus foram as espécies mais numerosas em seus estudos nas áreas de Cerrado. Essas mesmas espécies foram encontradas no presente trabalho no sul de Minas, podendo ser consideradas importantes para a cafeicultura da região do Cerrado. 
Tabela 4 - Análise faunística de ácaros da família Phytoseiidae encontrados em fragmentos florestais, adjacentes aos cafezais, em quatro coletas nos anos de 2004 e 2005 na região Sul de Minas, estado de Minas Gerais.

\begin{tabular}{|c|c|c|c|c|c|c|}
\hline Espécie & Número de espécimes & Número de coletas & $\mathrm{D}^{1}$ & $A^{2}$ & $\mathrm{~F}^{3}$ & $\mathrm{C}^{4}$ \\
\hline Amblyseius acalyphus & 3 & 1 & ND & $\mathrm{R}$ & $\mathrm{PF}$ & $\mathrm{Y}$ \\
\hline Amblyseius aerialis & 5 & 2 & ND & $\mathrm{R}$ & $\mathrm{PF}$ & $\mathrm{W}$ \\
\hline Amblyseius chiapensis & 1 & 1 & ND & $\mathrm{R}$ & $\mathrm{PF}$ & $\mathrm{Y}$ \\
\hline Amblyseius compositus & 57 & 4 & $\mathrm{D}$ & $\mathrm{C}$ & $\mathrm{F}$ & $\mathrm{W}$ \\
\hline Amblyseius herbicolus & 416 & 4 & $\mathrm{D}$ & $\mathrm{Ma}$ & MF & $\mathrm{W}$ \\
\hline Amblyseius hexadens & 2 & 1 & ND & $\mathrm{R}$ & $\mathrm{PF}$ & $\mathrm{Y}$ \\
\hline Amblyseius impressus & 49 & 4 & $\mathrm{D}$ & $\mathrm{C}$ & $\mathrm{F}$ & $\mathrm{W}$ \\
\hline Amblyseius operculatus & 84 & 3 & $\mathrm{D}$ & A & MF & $\mathrm{W}$ \\
\hline Amblyseius sp.1 & 4 & 2 & ND & $\mathrm{R}$ & $\mathrm{PF}$ & $\mathrm{W}$ \\
\hline Amblyseius sp.2 & 6 & 2 & $\mathrm{D}$ & $\mathrm{R}$ & $\mathrm{PF}$ & $\mathrm{W}$ \\
\hline Amblyseius spiculatus & 11 & 2 & $\mathrm{D}$ & $\mathrm{D}$ & $\mathrm{PF}$ & $\mathrm{W}$ \\
\hline Euseius alatus & 142 & 4 & $\mathrm{D}$ & $\mathrm{Ma}$ & MF & $\mathrm{W}$ \\
\hline Euseius citrifolius & 8 & 3 & $\mathrm{D}$ & $\mathrm{D}$ & $\mathrm{PF}$ & $\mathrm{W}$ \\
\hline Euseius concordis & 40 & 4 & $\mathrm{D}$ & $\mathrm{C}$ & $\mathrm{F}$ & $\mathrm{W}$ \\
\hline Euseius ho & 102 & 4 & $\mathrm{D}$ & $\mathrm{Ma}$ & $\mathrm{MF}$ & $\mathrm{W}$ \\
\hline Galendromus annectens & 2 & 1 & ND & $\mathrm{R}$ & $\mathrm{PF}$ & $\mathrm{Y}$ \\
\hline Iphiseiodes zuluagai & 9 & 3 & $\mathrm{D}$ & $\mathrm{D}$ & $\mathrm{PF}$ & $\mathrm{W}$ \\
\hline Iphiseiodes affs. neonobilis & 406 & 4 & $\mathrm{D}$ & $\mathrm{Ma}$ & $\mathrm{MF}$ & $\mathrm{W}$ \\
\hline Leonseius regularis & 256 & 4 & $\mathrm{D}$ & $\mathrm{Ma}$ & $\mathrm{MF}$ & $\mathrm{W}$ \\
\hline Neoseiulus affs. constrictatus & 9 & 1 & $\mathrm{D}$ & $\mathrm{D}$ & $\mathrm{PF}$ & $\mathrm{W}$ \\
\hline Neoseiulus constrictatus & 14 & 2 & $\mathrm{D}$ & $\mathrm{D}$ & $\mathrm{PF}$ & $\mathrm{W}$ \\
\hline Neoseiulus sp. & 2 & 1 & ND & $\mathrm{R}$ & $\mathrm{PF}$ & $\mathrm{Y}$ \\
\hline Neoseiulus tunus & 56 & 4 & $\mathrm{D}$ & $\mathrm{C}$ & $\mathrm{F}$ & $\mathrm{W}$ \\
\hline Phytoseiulus macropilis & 1 & 1 & ND & $\mathrm{R}$ & $\mathrm{PF}$ & $\mathrm{W}$ \\
\hline Phytoseius (Horridus) sp. & 69 & 4 & $\mathrm{D}$ & $\mathrm{C}$ & $\mathrm{F}$ & $\mathrm{W}$ \\
\hline Proprioseiopsis cannaensis & 3 & 2 & ND & $\mathrm{R}$ & $\mathrm{PF}$ & $\mathrm{W}$ \\
\hline Proprioseiopsis affs. neotropicus & 3 & 2 & ND & $\mathrm{R}$ & $\mathrm{PF}$ & $\mathrm{W}$ \\
\hline Proprioseiopsis dominigos & 1 & 1 & ND & $\mathrm{R}$ & $\mathrm{PF}$ & $\mathrm{Y}$ \\
\hline Silvaseius sp. & 1 & 1 & ND & $\mathrm{R}$ & $\mathrm{PF}$ & $\mathrm{Y}$ \\
\hline Typhlodromips sabaculus & 3 & 1 & ND & $\mathrm{R}$ & $\mathrm{PF}$ & $\mathrm{Y}$ \\
\hline Typhlodromips affs.sabaculus & 21 & 3 & $\mathrm{D}$ & $\mathrm{C}$ & $\mathrm{F}$ & $\mathrm{W}$ \\
\hline Typhlodromina sp. & 1 & 1 & ND & $\mathrm{R}$ & $\mathrm{PF}$ & $\mathrm{Y}$ \\
\hline Typhlodromips manihoti & 10 & 4 & $\mathrm{D}$ & $\mathrm{D}$ & $\mathrm{PF}$ & $\mathrm{W}$ \\
\hline Typhlodromips sp.1 & 31 & 2 & $\mathrm{D}$ & $\mathrm{C}$ & $\mathrm{F}$ & $\mathrm{W}$ \\
\hline Typhlodromips sp. 2 & 3 & 1 & ND & $\mathrm{R}$ & $\mathrm{PF}$ & $\mathrm{Y}$ \\
\hline Typhlodromalus limonicus & 7 & 1 & $\mathrm{D}$ & $\mathrm{R}$ & $\mathrm{PF}$ & $\mathrm{Y}$ \\
\hline Typhlodromus (Anthoseius) sp. & 2 & 2 & ND & $\mathrm{R}$ & $\mathrm{PF}$ & $\mathrm{W}$ \\
\hline
\end{tabular}

${ }^{1}$ Dominância : SD - superdominante, D - dominante, ND - não dominante.

(Método de Laroca e Mielke).

${ }^{2}$ Abundância : ma - muito abundante, a - abundante, c - comum, d - disperso, $r$ - raro.

${ }^{3}$ Frequência : $\mathrm{PF}$ - pouco frequente, $\mathrm{MF}$ - muito frequente, $\mathrm{F}$ - frequente.

${ }^{4}$ Constância : W - constante, $\mathrm{Y}$ - acessória, $\mathrm{Z}$ - acidental. 


\section{CONCLUSÕES}

Os ácaros predadores pertencentes à família Phytoseiidae mostraram-se dispersos nos ecossistemas naturais estudados, dando sua parcela de contribuição dentro da cadeia ecológica desses ambientes, bem como nos agroecossistemas cafeeiros adjacentes, podendo ocorrer uma migração natural dos ácaros entre os dois sistemas estudados. Pode-se, ainda, considerar que os agroecossistemas podem influenciar as áreas de vegetação natural, devendo-se promover formas efetivas de manter esses ambientes imunes de intervenção antrópica.

\section{AGRADECIMENTOS}

Ao Consórcio Brasileiro de Pesquisa e Desenvolvimento do Café - CBP\&D/Café pelo apoio financeiro e bolsa concedida e à FAPEMA e CNPq pela concessão de bolsas.

\section{REFERÊNCIAS BIBLIOGRÁFICAS}

ALTIERI, M.A. Agroecologia: bases científicas para uma agricultura sustentável. Guaíba: Agropecuária, 2002. 592 .

CASTRO, T.M.M.G.; MORAES, G.J. de. Mite diversity on plants of different families found in the brasilian atlantic forest. Neotropical Entomology, Londrina, v.36, n.5, p.774-782, 2007.

DAUD, R.D.; FERES, R.J.F. Diversidade e flutuação populacional de ácaros (Acari) em Mabea fistulifera Mart. (Euphorbiaceae) de dois fragmentos de Mata Estacional Semidecídua em São José do Rio Preto, SP. Neotropical Entomology, Londrina, v.34, n.2, p.191-201, 2005.

DEMITE, P.R.; FERES, R.J.F. Influência de vegetação vizinha na distribuição de ácaros em Seringal (Hevea brasiliensis Muell. Arg., Euphorbiaceae) em São José do Rio Preto, SP. Neotropical Entomology, Londrina, v.34, n.5, p.829-836, 2005.

DEMITE, P.R.; FERES, R.J.F. Ocorrência e flutuação populacional de ácaros 9Acari) associados a seringais (Hevea brasiliensis Muell. Arg.) vizinhos a fragmentos de Cerrado. Neotropical Entomology, Londrina, v.36, n.1, p.117-127, 2007.

FERES, R.J.F. et al. Ácaros (Acari, Arachnida) de plantas ornamentais na região noroeste do estado de São Paulo,
Brasil: inventário e descrição dos sintomas causados pelos fitófagos. Revista Brasileira de Entomologia, São Paulo, v.53, n.3, p.466-474, 2009.

FERES, R.J.F.; LOFEGO, A.C.; OLIVEIRA, A.R. Ácaros plantícolas (Acari) da "Estação Ecológica do Noroeste Paulista", Estado de São Paulo, Brasil. Biota Neotropica, São Paulo, v.6, n.1, p.1-14, 2005.

FERES, R.J.F.; MORAES, G.J. Phytoseiid mites (Acari: Phytoseiidae) from woody areas in the State of São Paulo, Brasil. Systematic and Applied Acarology, London, v.3, p.125-132, June 1998.

FERES, R.J.F.; ROSSA-FERES, D. de C.; DAUD, R.D.; SANTOS, R.S. Diversidade de ácaros (Acari, Arachinida) em seringueiras (Hevea brasiliensis Muell. Arg. , Euphorbiaceae) na região noroeste do estado de São Paulo, Brasil. Revista Brasileira de Zoologia, Curitiba, v.19, n.1, p.137-144, 2002.

\section{FLECHTMANN, C.H.W. Ácaros de importância agrícola. 6.ed. São Paulo: Nobel, 1985. 189p.}

HERNANDES, F.A.; FERES, R.J.F. Diversidade e sazonalidade de ácaros (Acari) em seringal (Hevea brasiliensis, Muell. Arg.) na região noroeste de São Paulo, Brasil. Neotropical Entomology, Londrina, v.35, n.4, p.523-535, 2006.

LOFEGO, A.C.; MORAES, G.J. de. Ácaros (Acari) associados a mirtáceas (Myrtacea) em áreas de Cerrado no estado de São Paulo com análise faunística das famílias Phytoseiidae e Tarsonemidae. Neotropical Entomology, Itabuna, v.35, n.6, p.731-746, 2006.

LOFEGO, A.C.; MORAES, G.J. de; McMURTRY, D. Three new species of phytoseiid mites (Acari: Phytoseiidae) from Brazil. Anais da Sociedade Entomologica do Brasil, Londrina, v.29, n.3, p.461-467, 2000.

LORENZI, H. Árvores brasileiras: manual de identificação e cultivo de plantas arbóreas do Brasil. 4.ed. Nova Odessa: Plantarum, 2002. 368p.

MCMURTRY, J.A.; CROFT, B.A. Life-styles of phytoseiid mites and their roles in biological control. Annual Review of Entomology, Berkeley, v.42, p.291-321, 1997. 
MORAES, G.J.; FLECHTMANN, C.H.W. Manual de acarologia: acarologia básica e ácaros de plantas cultivadas no Brasil. Ribeirão Preto: Holos, 2008. 308p.

PALLINI FILHO, A.; MORAES, G.J.; BUENO, V.H.P. Ácaros associados a cafeeiro (Coffea arabica $\mathrm{L}$.) no sul de Minas Gerais. Ciência e Prática, Lavras, v.16, n.3, p.303-307, 1992.

REIS, P.R.; SOUSA, E.O.; TEODORO, A.V.; PEDRO NETO, M. Effect of prey density on the functional and numerical responses of two species of predaceous mites (Acari: Phytoseiidae). Neotropical Entomology, Itabuna, v.32, n.3, p.461-467, July/Sept. 2003.

REIS, P.R.; TEODORO, A.V.; PEDRO NETO, M. Predatoty activity of phytoseiid on the development stages of coffee ringspot mite (Acari: Phytoseiidae, Tenuipalpidae). Anais da Sociedade Entomológica do Brasil, Itabuna, v.29, n.3, p.547-553, set. 2000.

REIS, P.R.; ZACARIAS, M.S. Ácaros em cafeeiro. Belo Horizonte: EPAMIG, 2007. 76p. (EPAMIG. Boletim Técnico, 81).

SPONGOSKI, S.; REIS, P.R.; ZACARIAS, M.S.

Acarofauna da cafeicultura de cerrado em Patrocínio, Minas Gerais. Ciência e Agrotecnologia, Lavras, v.29, p.9-17, 2005.

ZACARIAS, M.S.; MORAES, G.J. de. Phytoseiidae mites (Acari) associated with rubber trees and other Euphorbiaceous plants in southeastern Brazil. Neotropical Entomology, Londrina, v.30, n.4, p.570-586, 2001. 